\title{
Relevance of the Evaluation Criteria Used in Clinical Trials for Alzheimer's Disease
}

\author{
Philippe Truffinet ${ }^{1}$, Régis Bordet ${ }^{2}$, Joël Ménard ${ }^{3}$ and the participants in Round Table ${ }^{\circ} 1$, Giens XXIV ${ }^{\star}$ \\ 1 Sanofi Aventis, Antony, France \\ 2 EA 1046, Faculté de Médecine, Université de Lille II, Département Universitaire de Pharmacologie, Lille, France \\ 3 SPIM, Faculté de Médecine René Descartes, Université Paris V, Paris, France
}

Text received 15 october 2008; accepted 20 november 2008

\section{Keywords:}

Alzheimer;

evaluation;

clinical trial;

biomarker

\begin{abstract}
The roundtable 1 "Relevance of the evaluation criteria used in clinical trials for Alzheimer's disease" made reference to the guideline published by the EMEA (European Medicines Agency) in July 2008 on the development of new treatments for Alzheimer's disease (AD) and other dementias, and addressed principally two of the three indications listed in the guideline: symptomatic improvement and disease-modification (primary prevention was hardly discussed). The discussions focussed on two main aspects: improvement of the selection of patients in clinical trials and clinical evaluation and biomarkers. The following suggestions were made:
\end{abstract}

- Reinforce the interest for clinical trials at the early stages of AD (including prodromal stage), particularly for diseasemodifiers.

- Strengthen the research centers' expertise with biomarkers, in a perspective of subsequent use in clinical trials, either for the description of the patients included, or as part of selection criteria. Furthermore, ongoing intercenter validation studies, in France, of neuro-imaging and biomarker assays in CSF, are essential for preparing multicenter clinical trials.

- Facilitate the conduct of ancillary studies with biomarkers, grafted on clinical studies.

- Further develop the training and experience of raters with functional scales, which are now required as one of the two primary endpoints in pivotal clinical trials, and with the additional items of ADAS-cog (Alzheimer's Disease Assessment scale, Cognitive sub scale), which are useful for the early stages of AD.

- Improve knowledge of functional clinical scales by in depth analysis of available databases, through public/private collaborations.

- Improve knowledge of relationship between rating scales used in clinical trials and tools used in clinical practice (which are usually different), in order to provide supporting evidence for the interpretation of the clinical relevance of clinical trials results.

- Consider the potential needs of adaptation of rating scales to the societal changes, in particular for the evaluation of cognitive performance.

- Discuss the possibility of measures for extending data protection for candidate disease-modifiers, considering the negative impact for all players of the constraints of duration and the difficulties of clinical trials.

- Further discuss the ethics and acceptability of placebo-controlled monotherapy studies on durations of 6-9 months.

\section{Introduction}

The roundtable 1 "Relevance of the evaluation criteria used in clinical trials for Alzheimer's disease" was held in the current environment of multinational development of innovative molecules by pharmaceutical companies, and of academic research of new

\footnotetext{
* For a list of participants, see the end of the article.
}

therapeutic targets. The magnitude of the public health issue of Alzheimer's disease (AD) is well documented: highly frequent disease, which affects an increasing number of subjects because of the ageing of the population, and results not only in subjects' disability, but also in great burden on the family circle. ${ }^{[1,2]}$ Available drugs are still limited despite intensive research and development activity: three cholinesterase inhibitors and one blocker of the 
glutamate NMDA receptors. Furthermore, there are heated debates on the cost-benefit ratio, with controversies on the recommendations for reimbursement in some countries. The development of new treatments faces multiple difficulties, as testified by the number of recent failures, even at the advanced stage of pivotal trials in patients.

The importance of the roundtable was reinforced by the French governmental plan on AD, which aims at federating research in the country. Also there is a need of maintaining the attractivity of France for the conduct of clinical trials, where it has had a strong position in the past years. The roundtable made reference to the guideline published in 2008 by the EMEA for the treatment of Alzheimer's disease and other dementias. ${ }^{[3]}$ The analysis of the guideline was a thread for the roundtable, and the changes from the previous 1997 EMEA guideline ${ }^{[4]}$ were highlighted. The roundtable addressed principally two of the three indications listed in the guideline: symptomatic improvement and disease-modification (primary prevention was hardly discussed). The discussions focussed on two main aspects: improvement of the selection of patients in clinical trials and clinical evaluation and biomarkers.

\section{Evolving guidelines}

Regulatory agencies gave a framework to clinical development of new treatments for AD through recommendations since the 90s. The FDA (Food and Drug Administration) published a guideline in $1990,{ }^{[5]}$ which remained at a draft stage, in which was introduced the requirement of two primary endpoints: cognitive, the expected target being cognitive function; and global or functional, to evidence the clinical relevance of the effect: "To gain an antidementia indication for a product, a sponsor must provide substantial evidence that the product 1 ) has a clinically meaningful effect and 2) exerts its effect on the 'core' manifestations of dementia. This compound requirement can be met by showing, in more than one adequate and well controlled clinical investigation, that the drug product is superior to an appropriate control treatment on both 1) a global assessment performed by a skilled clinician and 2) a performance based, objective test instrument providing a comprehensive assessment of cognitive functions." The EMEA, in its guideline dated 1997, adopted again the principle of co-primary endpoints, and broadened the scope of evaluations, by adding the request of a functional evaluation, which may be considered one of the two primary endpoints, as an alternative to the global evaluation. The most recent European guideline, finalized in 2008, kept the principles of the previous one and expanded the arguments (it has grown from 7 to 19 pages), while bringing a few evolutions. It is now specified that the second primary endpoint (in addition to the cognitive endpoint) must be the functional evaluation (the global evaluation becomes a secondary endpoint). The behavioural disorders were considered separately, being usually used as secondary evaluations to further characterize the therapeutic effect, as recommended in the guideline.

The definition of the indications got more precise with the evolution of guidelines. The FDA guideline kept focussed on treatments now qualified "symptomatic" for the mild to moderate severity stages of dementia, based on the experience available at this point in time. Indications were defined in the $1997 \mathrm{Eu}-$ ropean Guideline as follows: "Symptomatic improvement, which may consist in enhanced cognition, more autonomy and/or improvement in neuropsychiatric and behavioural dysfunction. Disease modification with slowing or arrest of symptom progression of the dementing process. For regulatory purposes a disease modifying effect will be considered when the pharmacologic treatment delays the underlying pathological or pathophysiological disease processes and when this is accompanied by an improvement of clinical signs and symptoms of the dementing condition. Primary prevention of disease by intervention in key pathogenic mechanisms at a pre-symptomatic stage." Regarding disease modifying drugs, it is now specified that "convincing" results on biomarkers a "convincing package of biological and/or neuroimaging data, e.g. showing delay in the progression of brain atrophy" should be obtained.

The field of indications became broader with guideline progression. The guidelines of the 90s were focussed on dementia of Alzheimer's type, whereas the 2008 guideline addressed other dementias. Furthermore, the interest of early diagnosis and treatment was acknowledged in this latest guideline. Reservations were made toward the concept of MCI (Mild Cognitive Impairment), considered too heterogeneous, but current researches on early diagnosis were mentioned in a positive way: "Currently epidemiological and clinical studies are underway to establish validated criteria for definition of "pre-dementia stages", and the Research Criteria proposed by a multinational workshop ${ }^{[6]}$ are listed in reference.

\section{Optimizing the selection of patients in clinical trials}

The diagnosis of $\mathrm{AD}$ at a stage of dementia is made on the DSM IV criteria of dementia of Alzheimer's type, and on the NINCDS-ADRDA (National Institute of Neurological and Communicative Disorders and Stroke-Alzheimer's Disease and Related Disorders Association) criteria for $\mathrm{AD}$, which are essentially clinical. The use of non-clinical information in these criteria is limited to the exclusion of differential diagnosis. 
However, the specificity of these criteria is unsatisfactory, with estimates of frequency of wrong diagnosis ranging from 23 to $88 \% .{ }^{[6]}$ The numerous recent researches on biomarkers ${ }^{[7,8]}$ showed that significant improvements in sensitivity and specificity can be obtained, principally through (i) lumbar puncture, which allows to quantify levels of $\mathrm{A} \beta 1-42$, d'A $\beta 1-40$, truncated forms of $A \beta$, total tau and phosphorylated tau, and (ii) neuroimaging, by MRI (Magnetic Resonnance Imagerie) which evidences hippocampal atrophy and the presence of absence of vascular lesions (white matter lesions, silence infarctus or micro-bleeding...), or by FDG PET-scan (Fluoro-Desoxy-Glucose Postiron Emission Tomography-scan) showing parieto-temporal hypometabolism. Studies also showed the interest of radio-labelled ligands of amyloid plaques, but their current use is still limited. Research criteria have been proposed, which use a selection of biomarkers as supporting diagnosis criteria. ${ }^{[6]}$

The use of biomarkers in entry checkup in clinical trials is usually relatively limited, in ongoing trials (information posted on the website Clinicaltrial.gov (http://www.clinicaltrials.gov/) as well as in trials recently published and/or presented in conferences. The phase II and phase III situations need to be distinguished. In phase III, the patients' selection classically aims at getting a representative sample, with respects to the target indication, and criteria therefore reflect clinical practice. Technologies not used in clinical practice would therefore not be appropriate in this perspective. However, biomarkers may allow to provide additional objective evidence of the diagnosis. It is in phase II that the use of biomarkers may be of greatest interest, in the aim of enriching selection of a sample most appropriate to evidence a therapeutic effect. The roundtable suggested that research centers use biomarkers on a systematic basis, in order to strengthen their expertise, in a perspective of subsequent use in clinical trials, either for the description of the patients included, or as part of selection criteria.

Genotyping, in particular of apolipoprotein E, is to be considered separately. It belongs more and more to the entry evaluations in clinical trials, usually not for selecting the patients but for assessing the impact of various genotypes on the therapeutic response. This use has been reinforced by the publication of apparent differences in therapeutic responses in genotype subgroups, in studies of rosiglitazone, ${ }^{[9]}$ and bapineuzumab (study presented at the ICAD 2008 conference).

\section{Study design, placebo and treatment duration}

The discussions addressed principally two difficulties in the design of AD studies: acceptability of placebo and duration of disease-modifier studies. There is a conflict between the need of performing placebo-controlled studies of sufficient duration and their acceptability, principally regarding symptomatic treatments for which monotherapy studies are conducted (in the case of disease-modifiers, studies are performed in add-on conditions). Placebo effects potentially interfering with evaluation of treatment effect were reported with increased frequency in the past years, in particular when treatment duration was reduced to less than 6 months. On the other hand, there is no consensus in the medical community on the potential loss of chances for the patients for not prescribing available drugs on a prolonged period. In France, the 6-month duration, which is recommended for pivotal studies by the EMEA guideline, has often been considered unacceptable by investigators and ethics committees. In contrast, authors like L. Schneider, in the USA, defended the use of placebo on this duration. ${ }^{[10]}$ The roundtable suggested that the position of the French investigators might be reconsidered in light of the recent evolutions of the environment.

The duration of clinical trials in the case of disease-modifiers, which was often approximately 1 year in the 90 s, is now frequently longer, usually 18 months, in line with the recommendations from the EMEA. However, it seems, from a few recent experiences, that the slopes of evolution might be smaller that in the past, with in particular a slower progression in the first 6 to 9 months, as in the case of the study of tarenflurbil presented at the ICAD conference in 2008. It was stated that the constraints of duration, associated to the high risk of failed studies, could lead to decrease in drug development in the indication despite the magnitude of medical needs, and it was suggested that measures for extending data protection might be appropriate in this context.

\section{Evaluation criteria}

Two main points were addressed: the relevance of clinical evaluation criteria, and their condition of use, and the biomarkers to be selected for clinical trials. Of note, the outcome criteria were reviewed in depth by a working group gathered by the European Alzheimer Disease Consortium (EADC). ${ }^{[11]}$

There is an ongoing debate on the sensitivity of ADAScog, which has become the standard tool for cognitive evaluation in clinical trials, and the potential interest of alternative instruments, more recently developed, such as the NTB battery. ${ }^{[12]}$ The claimed advantages of this battery over ADAS-cog are the evaluation of executive functions, and a claimed better sensitivity at early stages of the disease. However, the roundtable participants commented that ADAS-cog remains an instrument with well established sensitivity to pharmacological effects, and that there is currently no strong evidence of the potential superiority of other 
instruments. The results of the phase II study of bapineuzumab presented at the ICAD 2008 conference did not show a greater sensitivity of NTB versus ADAS-cog. They expressed their interest in the new items of ADAS-cog, and recommended trainings to these items which are currently less well known than the "core" items. Besides, ADAS-cog is a research tool which is not used in clinical practice. Because of this gap, there is a need to better document the clinical relevance of ADAS-cog in clinical trials, eg by studies of correlations between various instruments.

The functional evaluation is currently based principally on two scales: ADCS-ADL ${ }^{[14]}$ and DAD. ${ }^{[15]}$ These scales have been used so far as secondary endpoints, and as such have not been worked upon as much as the cognitive and global evaluations. Their variability in the current conditions of use is greater than that of global scales. It has therefore been suggested by the roundtable that validation and training activities be conducted in the French research centers, as previously done for ADAS-cog. Furthermore, it has been suggested to use available databases for in depth analysis of the properties of the scales. Of note, the concept of responders has not been considered appropriate, because a dichotomy is introduced, on an arbitrary and debatable threshold, for distributions which are rather continuous in the case of AD.

Biomarkers should have been shown to progress over time for being used as endpoints in clinical trials. This demonstration has been done so far for volumetric imaging, ie global and hippocampal volumetry as measured by MRI and FDG PET. Only MRI volumetry has been used so far in large pivotal trials. The potential interest of other markers depend on the mechanisms of action studied, eg markers of the amyloid cascade for an action on this cascade. Ongoing intercenter validation studies, in France, of neuro-maging and biomarker assays in CSF are essential for preparing multicenter clinical trials. Of note, cohort studies such as ADNI (followed by European and Japanese ADNI), which aim at providing multicenter biomarker data, have not been discussed at the roundtable. Furthermore, clinical studies might provide a support for researches on biomarkers, through ancillary studies.

\section{Participants}

Serge Bakchine (CHU Reims), Luc Buée (INSERM U 422, Lille), Soraya Cherchali (Sanofi Aventis R\&D), Maylis Coste (IRI Servier), Bruno Dubois (Fédération Neurologie 4, Groupe Hospitalier Pitié Salpétrière, Paris), Françoise Duveau (Laboratoire ESAI), Jean-Marie Goehrs (Merck Sharp \& Dohme Chibret), Sylvia Goni (Laboratoires lundbeck), Philippe Gustovic (Wyeth Pharmaceuticals), Catherine Lassale (LEEM Recherche),
Jean-Marc Orgogozo (CHU Bordeaux), Florence Pasquier (CHU Lille), Odile Regnier (CNAMTS), Franck Semah (CHU Lille), Jacques Touchon (Neurologie B, CHU Montpellier), Marc Verny (Centre de Gériatrie, Groupe Hospitalier Pitié Salpétrière, Paris), Mohammed Zaïm (Laboratoire Pierre Fabre).

\section{References}

1. Cummings JF. Alzheimer's disease. N Engl J Med 2004; 351(1): 56-67

2. Morris JC. Dementia update 2005. Alzheimer Dis Assoc Disord 2005; 19 ; 100-17

3. CHMP 2008. Guideline on medicinal products for the treatment of Alzheimer's disease and other dementias. http://www.emea.europa. eu/pdfs/human/ewp/055395en.pdf

4. EMEA. Note for guidance on medicinal products in the treatment of Alzheimer's disease. 1997. http://www . emea. europa.eu/

5. FDA. Guidelines for the clinical evaluation of antidementia drugs, first draft, November 8, 1990. http: //www . fda.gov

6. Dubois B, Feldman HH, Jacova C, et al. Research criteria for the diagnosis of Alzheimer's disease: revising the NINCDS-ADRDA criteria. Lancet Neurol 2007; 6: 734-46

7. Shaw LM, Korecka M, Clark CM, et al. Biomarkers of neurodegeneration for diagnosis and monitoring therapeutics Nature reviews. Drug Discovery 2007; 6: 295-303

8. Blennow K. Cerebrospinal fluid protein biomarkers for Alzheimer's disease. NeuroRx 2004: 1: 213-25

9. Risner ME, Saunders AM, Altman JFB, et al. Efficacy of rosiglitazone in a genetically defined population with mild-to-moderate Alzheimer's disease. Rosiglitazone in Alzheimer's disease study group. The Pharmacogenomics Journal 2006; 6, 246-54

10. Schneider L. AD2000: donepezil in Alzheimer's disease. Lancet 2004; 363: 2100-1

11. Vellas B, Andrieu S, Sampaio C, et al. Endpoints for trials in Alzheimer's disease: a European task force consensus Lancet Neurol 2008; 7: 436-50

12. Harrison J, Minassian SL, Jenkins L, et al. A neuropsychological test battery for use in Alzheimer disease clinical trials. Arch Neurol 2007; 64(9): 1323-9

13. Mohs RC, Knopman D, Petersen RC, et al. Development of cognitive instruments for use in clinical trials of antidementia drugs: additions to the Alzheimer's disease assessment scale that broaden its scope: the Alzheimer's disease cooperative study. Alzheimer Dis Assoc Disord 1997; 11(suppl 2): S13-S21

14. Galasko D, Bennett D, Sano M, et al. A inventory to assess activities of daily living for clinical trials in Alzheimer's disease. Alzheimer Dis Assoc Disord 1997; 11 (Suppl 2): 533-9

15. Gauthier S, Gelinas I, Gauthier L. Functional disability in Alzheimer's disease. Int Psychogeriatr 1997; 9 (Suppl 1): 163-5

Correspondence and offprints: Philippe Truffinet, Sanofi Aventis, Investigations Cliniques, Neurologie, 20 rue Raymond Aron, 92165 Antony Cedex, France.

E-mail: philippe.truffinet@sanofi-aventis.com 